\title{
Comparison Between Follicular Antimüllerianhormonelevels Between Women's Withpolycystic Ovarian Syndrome And Normal Ovulatory Women Undergoing Intracytoplasmic Sperm Injection (ICSI) Techniques.
}

\author{
Inam Abdul- Wahid ${ }^{1}$, Affan E Hassan², Basil O Saleh ${ }^{3}$ and \\ ZeyadTareq Abdul Razzaq ${ }^{4}$ \\ ${ }^{1} M . B . C h . B, M S c$. \\ ${ }^{2}$ M.B.Ch.B, MSC. Ph.D \\ ${ }^{3}$ M.B.Ch.B, MSC. Ph.D \\ ${ }^{4}$ M.B.Ch.B, MSC.Ph.D
}

\begin{abstract}
Background: $A M H$ is a glycoprotein of the transforming growth factor- $\beta$ (TGF- $\beta$ ) superfamily; it has as an important role in regulation of the ovarian follicle development.AMH Produced by granulosa cells of growing follicles after birth.Polycystic ovary syndrome (PCOS) is the most common cause of menstrual dysfunction, infertility, and hyperandrogenism, and itsprevalence is about 5-10\% in female population of reproductive-age women. Follicular fluid $(F F)$ surrounding the oocyte may play an important role in determining oocyte quality and the future potential to achieve fertilization and development of embryo and it provides a very good environment for the development of the oocytes.

Objective: Compare the level of follicular fluid of anti-mellerian hormone (AMH) in women with PCOS and normal ovulatory women that undergoing intra cytoplasmic sperm injection (ICSI) techniques. And in prediction of clinical outcome (pregnancy) of intra cytoplasmic sperm injection (ICSI) in women with PCOS by measuring the cut-off value of FF of AMH levels.

Subjects and Methods: This case-control study was carried out at College of Medicine, University of Baghdad and at Kamal Al-Samarai Hospital, Baghdad, Iraq, during the period from December 2014 to February 2016. The study involved 23 women with PCOS and 22 healthy women (male factor). Follicular fluid samples were collected from all women on day of oocyte pick up by U/S guidedtransvaginal technique and used for measurements of the concentrations of AMH. ELISA technique was used for hormone estimations.

Results: The results of this study revealed that follicular fluid $(F F)$ concentrations of AMH were significantly increased in PCOS women when compared to healthy women $(P=0.034)$. The cut-off values of FF of AMH levels for prediction of pregnancy of ICSI in PCOS women were found to be $110.17 \mathrm{ng} / \mathrm{ml}$. In healthy women with normal ovulatory cycle, the cut-off values of FF levels of AMH were found to be $85.46 \mathrm{ng} / \mathrm{ml}$.

Conclusion: This study revealed the clinical importance of the measurements of FF levels of AMH in prediction of cases of PCOS in women's undergoing ICSI techniques.
\end{abstract}

Key Words: AHM, PCOS, follicular fluid, ICSI.

\section{Introduction}

Polycystic ovarian syndrome (PCOs) considered as the most common endocrine disorder in women in reproductive age with a prevalence of about $5-10 \%$ in female populationand it is considered as the most common cause of chronic anovulation and hyperandrogenism in young women (1). PCOS is a diagnosis that is being made more commonly in adolescent girls; mirroring the increasing rate of obesity in this population (2). The exact mechanism affecting ovarian steroid genesis in PCOS is still unclear. Insulin resistance is a common feature of PCOS that has been observed (3). Hyper insulinemia has been recognized as a contributory factor for ovarian disruption due to increased production of ovarian androgens (4). Also obesity is commonly seen associated with this disorder; this further worsens thestate of insulin resistance state in thosepatients. This heterogeneous nature of the disease has led to difficulty in building consensus for the standard diagnostic criteria (5). National Institute of Health (NIH) (1990) suggested the presence of oligomenorrhea with clinical or biochemical hyperandregenimia are essential for the diagnosis (6). The Rotterdam Consensus (2003) expanded the diagnostic criteria for PCOS by adding the antral follicle count (AFC) on ultrasound as one of the diagnostic criterion and recommended that at least two out of the three criteria should be present (7). Finally, the Androgen Excess Society (2006) recommended that to define PCOS patients (the patient must have clinical and biochemical features of the disorder, these include: clinical and/or biochemical evidence of excess in androgen levels with either oligomenorrhea and/or polycystic ovarieson pelvic ultrasound)(8). 
Excessive ovarian production of Anti-Müllerian Hormone (AMH), secreted by growing follicles, is now considered as an important feature of PCOS. Serum AMH is synthetized by small antral follicles of the ovaries, which are seen on ultrasound. Serum AMH could therefore be used as a representative for the AFC in the diagnosis of PCOS. Serum AMH has also demonstrated its utility in the treatment of infertility. But the absence of an international standard for serum AMH assay and the inability to define thresholds makes application of serum AMH more difficult (9).

Increased AMH levels correlated with PCOS severity and are associated with greater ovarian stimulation and higher clinical pregnancy rates following assisted reproductive technology. These data characterize a population of women with elevated AMH levels, demonstrating that the vast majority of women with AMH >10 ng/mL have PCOS (10).It has been founded that higher AMH levels in PCOS women compared with normo-ovulatory controls matched for age and BMI. However, these levels decreased at a higher rate over time in PCOS patients, narrowing the difference. The estimated reproductive lifespan of PCOS cases was extended by 2 years on average compared with normal ovulatory women(11).

\section{Subjects And Methods:}

This study involved 65 women aged nearly $15-42$ year and classified into two groups; 33 women with PCOS and 32 women with normal ovulation [male factor infertility]. Both groups of women will undergo hormonal stimulation according to ICSI technique at Kamal AL -Samarai Hospital and Baghdad IVF center. The index of obesity [BMI], history of cycle regularity, hirsutism, acne, number of children was recorded. Women who have had chronic endocrine disorder were excluded from the study.

Blood sample collected from peripheral vein of each woman on the day of the oocyte pick-up. Follicular fluid was taken from each woman from the pool of ovarian follicles guided by transvaginal ultrasound technique. AMH level measured in follicular fluid using ELISA technique. The FF levels of AMH in PCOS women and women's with normal ovulation were measure and compared during ICSI technique. The differences in the mean $( \pm \mathrm{SE})$ values of hormonal study were calculated.

\section{Statistical Analysis}

In our study the data of the 65 patients were analyzed by the statistical package for social science (SPSS) software version 17.

Descriptive statistics were presented as mean, standard error, frequencies and percentage.

The comparisons of follicular fluid AMH were tested using paired t test.The comparison between PCOs with nonPCOs was done using students's t-test.In each group, the student t-test was used to compare the parameters of the pregnant versus thenon pregnant patients. ROC curve for the follicular fluid AMH were used toexpress the sensitivity and specificity index of the follicular fluid AMH. A level of significance ( $p$ value) of $\leq 0.05$ was considered significant.

\section{Results}

The clinical characteristics of the studied groups are shown by table (1). The mean values of age of PCOs women's $(30.43 \pm 1.28)$ did not differ significantly from that of control group women's $(30.40 \pm 1.25)$. The mean valuesof BMI did not differ significantly between the PCOsand control groups women's $(26.2 \pm 0.414$, $25.2 \pm 0.598$, respectively). The female age of both study groups was ranged between 19-42years. The BMI of both studied groups was ranged between 35.35- 49.39.

Table (1): General demographic data among studied groups

\begin{tabular}{|l|l|l|l||}
\hline Parameter & $\begin{array}{l}\text { PCOs } \\
\text { M } \pm \text { SE }\end{array}$ & $\begin{array}{l}\text { Non PCOs } \\
\text { M } \pm \text { SE }\end{array}$ & P Value \\
\hline \hline Age & $30.43 \pm 1.28$ & $30.40 \pm 1.25$ & 0.831 \\
\hline \hline BMI & $26.2 \pm 0.414$ & $25.2 \pm 0.598$ & 0.173 \\
\hline
\end{tabular}

PCOs: Polycystic ovary syndrome patients. M \pm SE: Mean \pm Standard Error.BMI: Body Mass Index.

Table (2) revealed that the mean \pm standard values of follicular fluid ( FF) AMH level of PCOs women's $(45.03 \pm 6.63)$ was significantly higher than that of non PCOs women's $(26.52 \pm 5.17, \mathrm{P}=0.034)$. 
Table (2): The values of follicular fluid level of AMH in PCOs and non PCOs groups.

\begin{tabular}{|l|l|l||l||}
\hline Parameter & $\begin{array}{l}\text { PCOs } \\
\text { M } \pm \text { SE }\end{array}$ & $\begin{array}{l}\text { Non PCOs } \\
\text { M } \pm \text { SE }\end{array}$ & P Value \\
\hline \hline AMH $(\mathrm{ng} / \mathrm{ml})$ & $\mathbf{4 5 . 0 3 \pm 6 . 6 3}$ & $\mathbf{2 6 . 5 2} \pm 5.17$ & $\mathbf{0 . 0 3 4}$ \\
\hline
\end{tabular}

* Significant $\mathrm{P}$ value $(\mathrm{P} \leq 0.05)$.

Table (3) showed the cutoff values of the follicular fluid AMH concentration for prediction of pregnancy outcome by using the percentiles 90 and 95 in PCOs, non PCOs and all patients in the study. This study may help in prediction of clinical outcome (pregnancy).AMH level were directly correlated with clinical pregnancy outcome. Using non pregnant (PCOs and non PCOs) in general to introduce a cutoff level for prediction of pregnancy outcome.

Table (3): Cutoff values of the follicular fluid AMH concentration for prediction of pregnancy outcome

\begin{tabular}{|l|l|l|}
\hline Parameter & AMHf(ng/ml) \\
\hline \hline \multirow{2}{*}{$\begin{array}{l}\text { PCOs } \\
\text { (non pregnant) }\end{array}$} & Percentiles 90 & 110.17 \\
\cline { 2 - 3 } & Percentiles 95 & 111.70 \\
\hline \hline \multirow{2}{*}{$\begin{array}{l}\text { Non PCOs } \\
\text { (non pregnant) }\end{array}$} & Percentiles 90 & 85.46 \\
\hline & Percentiles 95 & 97.16 \\
\hline \hline \multirow{2}{*}{$\begin{array}{l}\text { All cases } \\
\text { (non pregnant) }\end{array}$} & Percentiles 90 & $\mathbf{9 9 . 5 1}$ \\
\cline { 2 - 3 } & Percentiles 95 & 110.25 \\
\hline
\end{tabular}

Figure (1) shows the ROC curve for the follicular fluid AMH. This figure revealed that the sensitivity and specificity index of follicular fluid AMH (70.3\%).

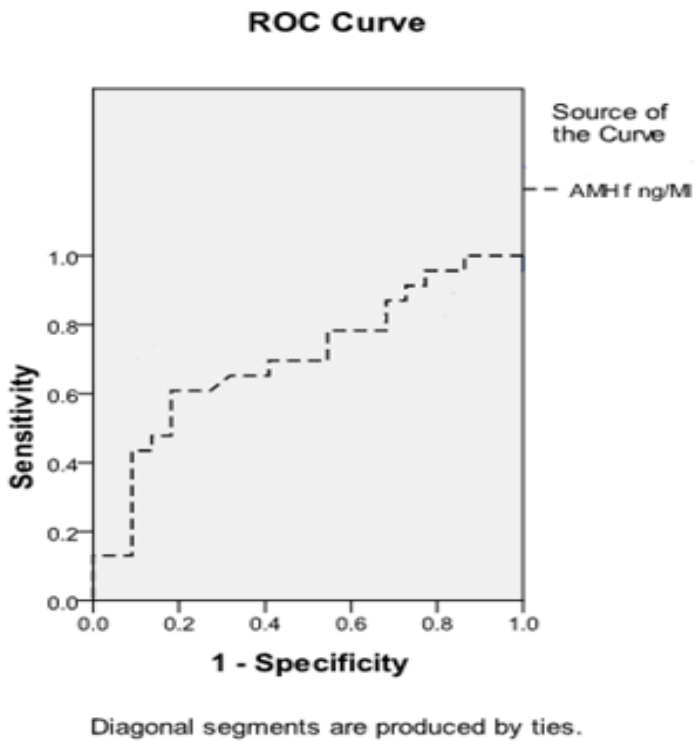

Figure (1): Receiver operating characteristic (ROC) curve analysis calculation of follicular fluid AMH

\section{Discussion}

In the present study female age of all study groups ranged between 19- 42 years. The mean values of age in the PCOs women's $(30.43 \pm 1.28)$ did not differ significantly from that of women's with normal ovulatory cycle $(30.40 \pm 1.25)$. This result is in agreement with result of Bukman\&Heineman, that suggested that although the age factor is an important in cases of infertility, it was not very precise in predicting reproductive potential (19). The mean value of BMI was not differing significantly between the women's with PCOs (26.2 \pm 0.414$)$ and control women's normal ovulatory cycle $(25.2 \pm 0.598)$. Reduced fertility in the obese women (BMI $\geq 30)$ is usually related to many factors including the deviation in endocrine and metabolic functions that can affect the follicular growth, implantation and clinical pregnancy outcome (20).

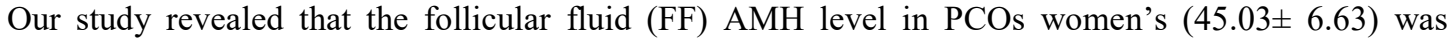
significantly higher than in the non PCOs women's $(26.52 \pm 5.17, \mathrm{P}=0.034)$. This result in agreement with study 
done by Cook et al, they notice that the PCOS women have a high level of AMH in the serum and follicular fluid than in other healthy controls women's (14). Moreover, it was founded that there was a significant correlation between the follicular fluid and serum concentrations of AMH in the PCOS group but not in controls (21). The ROC curve for follicular fluid AMH, this curves were constructed by computing the sensitivity and specificity of the follicular fluid AMH, in this study area under the curve consider high for FF AMH. Low level of anti-Müllerian hormone predicts a low response to ovarian hyperstimulation programs, while a greater level may predicts a high response (22). Subsequently, higher AMH levels are usually associated with greater chance of live birth after IVF, even after adjusting for age (23). Means that the AMH is a good predictor of ovarian response.

The sensitivity and specificity index of the follicular fluid AMH (70.3\%). This result in agreement with study done by Alipour et al, that study the sensitivity of AMH and FSH serum level, the result of the study indicate that AMH has high sensitivity. AMH seems to be more useful in the early diagnosis of premature ovarian failure (24). The results done by Krawczuk-Rybak et al revealed that the AMH measurement can be used as a sensitive marker of a reduced ovarian reserve in young cancer survivors (25).

The main finding of this study is that the role of AMH to diagnose PCOS, especially when combined with the other diagnostic criteria of PCOS. So, AMH can be substituted for PCO treatment and is equally good in diagnosing PCOS (20). Data about our control group agree with previous reports from Fanchin et al. (21) who found a tight relationship between the AMH serum level and the FN at U/S in regularly menstruating infertile women studied at baseline before undergoing in vitro fertilization. Van Rooij et al. (16) also showed that the AMH level was a good predictor of the number of retrieved oocytes, as previously reported by Seifer et al. (17). However, our data have produced new information by showing that the 6- to 9-mm follicles do not contribute to the serum AMH level in normo-ovulatory women, whereas being overweight has a mild, although significant, negative influence on this parameter.

\section{Conclusion}

This study revealed the clinical importance of the measurements of FF levels of AMH in prediction of cases of PCOS in women's undergoing ICSI techniques. Also our study revealed the clinical importance of the measurements of the FF levels of AMH in assessment of clinical outcome or pregnancy of ICSI in PCOS infertile women.The study revealed that the serum AMH is a good predictor of ovarian response. In our study we found that AMH directly correlated with the clinical pregnancy outcome. The obtained cut-off values using the non-pregnant (PCOs and non PCOs) in general of serum and FF concentrations of AMH are very important for prediction of the result of the ICSI technique.

\section{References}

[1]. Knochenhauser ES, Key TJ, KahserMM, Waggoner W, Boots LR, et al. (1998) Prevalence of polycystic ovary syndrome in unselected black and white women of the southeastern United States: a prospective study. J ClinEndocrinolMetab 83:3078-3082.

[2]. Anderson AD, Solorzano CM, McCartney CR (2014) Childhood obesity and its impact on the development of adolescent PCOS.SeminReprod Med 32: 202-213.

[3]. Dunaif A, Segal KR, Futterweit W, Dobrjansky A (1989) Profound peripheral insulin resistance, independent of obesity, in polycystic ovary syndrome. Diabetes 38:1165-1174.

[4]. DiamantiKE, DunaifA (2012) Insulin resistance and the polycystic ovary syndrome revisited: an update on mechanisms and implications.Endocr Rev 33: 981-1030.

[5]. Sheehan MT (2004) Polycystic ovarian syndrome: diagnosis and management.Clin Med Res 2: 13-27.

[6]. Zawadzki J, Dunaif A (1992) Diagnostic criteria for polycystic ovary syndrome: towards a rational approach. Polycystic ovary syndrome. Blackwell Scientific Publications, Cambridge, USA.

[7]. Rotterdam ESHRE/ASRM-Sponsored PCOS Consensus Workshop Group (2004) Revised 2003 consensus on diagnostic criteria and long-term health risks related to polycystic ovary syndrome.FertilSteril 81: 19-25.

[8]. Azziz R, Carmina E, Dewailly D, Diamanti-Kandarakis E, Escobar-Morreale HF, et al. (2009) The Androgen Excess and PCOS Society criteria for the polycystic ovary syndrome: the complete task force report.FertilSteril 91: 456-488.

[9]. Dumont A, Robin G, Catteau-Jonard S and Dewailly D (2015): Role of Anti-Müllerian Hormone in pathophysiology, diagnosis and treatment of Polycystic Ovary Syndrome: Reproductive Biology and Endocrinology, 201513:137.

[10]. Tal R, Seifer DB, Khanimov M, Malter HE, Grazi RV, Leader B (2014):Characterization of women with elevated antimüllerian hormone levels $(\mathrm{AMH})$ : correlation of $\mathrm{AMH}$ with polycystic ovarian syndrome phenotypes and assisted reproductive technology outcomes.Am J Obstet Gynecol. 2014 Jul;211(1):59.e1-8. doi: 10.1016/j.ajog.2014.02.026.

[11]. Tehrani FR, Solaymani-DodaranM,Hedayati M, and AziziF(2010):Is polycystic ovary syndrome an exception for reproductive aging? Human Reproduction, Vol.25, No.7 pp. 1775-1781, 2010.

[12]. Van Rooij IA, Broekmans FJ, TeVelde ER, Fauser BC, Bancsi LF, Jong FH and Themmen AP (2002): Serum AMH levels: a novel measure of ovarian reserve. Hum Reprod 17:3065-3071.

[13]. Seifer DB, MacLaughlin DT, Christian BP, Feng B and Shelden RM F (2002): Early follicular serum Mullerian-inhibiting substance levels are associated with ovarian response during assisted reproductive technology cycles. FertilSteril 77:468-471.

[14]. Cook CL, Siow Y, Brenner AG, Fallat ME 2002 Relationship between serum Mullerian-inhibiting substance and other reproductive hormones in untreated women with polycystic ovary syndrome and normal women. FertilSteril 77:141-146.

[15]. Eilertsen TB, Vanky EandCarlsen SM. (2012): Anti-Mullerian hormone in the diagnosis of polycystic ovary syndrome: can morphologic description be replaced? Human Reproduction, Vol.27, No.8 pp. 2494-2502, 2012 Advanced Access publication on June 12, 2012 doi:10.1093/humrep/des213. 
[16]. Fanchin R, Schonauer LM, Righini C, Guibourdenche J, Frydman R, Taieb J 2003 Serum AMH is more strongly related to ovarian follicular status than serum inhibin B, estradiol, FSH and LH on day 3. Hum Reprod. 18:323-327.

[17]. Davoodi, Salsabili, Sadeghipour(2005):Effects of Human Follicullar Fluid and Synthetic Serum Substitute on Human Embryonic Development and Cell Cleavage.AcataMedica Iranica.2005;43(1): 1-6.

[18]. Revelli A, Piane LD, Casano S, Molinari E, Massobrio M, and Rinaudo P (2009): Follicular fluid content and oocyte quality: from single biochemical markers to metabolomics.ReprodBiolEndocrinol. 2009; 7: 40.Published online 2009 May 4. doi: 10.1186/14777827-7-40.

[19]. Bukman A and Heineman MJ (2001): ovarian reserve testing and the use of prognostic models in patients with subfertility.HumRuprod update.2001; 7: 581-590.

[20]. Fedorcsak P, Olav Dale and P Storeng R (2004): impact of overweight and underweight on assisted reproduction treatment. Hum Reprod. 2004; 19:2523-2525.

[21]. Das M1, Gillott DJ, Saridogan E, Djahanbakhch O (2008): Anti-Mullerian hormone is increased in follicular fluid from unstimulated ovaries in women with polycystic ovary syndrome. Hum Reprod. 2008 Sep; 23(9):2122-6. doi: 10.1093/humrep/den185. Epub 2008 Jun 10.

[22]. La Marca A and Sunkara SK (2013): "Individualization of controlled ovarian stimulation in IVF using ovarian reserve markers: from theory to practice". Human Reproduction Update 20 (1): 124-40. doi:10.1093/humupd/dmt037. PMID 24077980.

[23]. Broer SL, Broekmans FJ, Laven JS and Fauser BC (2014): "Anti-Müllerian hormone: ovarian reserve testing and its potential clinical implications". Human Reproduction Update 20 (5): 688-701. doi:10.1093/humupd/dmu020. PMID 24821925.

[24]. Alipour F, Rasekhjahromi A, Maalhagh M, Sobhanian S, 3 and Hosseinpoor M (2015): Comparison of Specificity and Sensitivity of AMH and FSH in Diagnosis of Premature Ovarian Failure. Published online 2015 May 31. doi: 10.1155/2015/585604. Journal ListDis Markers; 2015PMC4465760).

[25]. Krawczuk-Rybak M, Leszczynska E, Poznanska M, Zelazowska-Rutkowska B, and Wysocka J (2013): Anti-Müllerian Hormone as a Sensitive Marker of Ovarian Function in Young Cancer Survivors. Int J Endocrinol. 2013; 2013: 125080.Published online 2013 Dec 15. doi: 10.1155/2013/125080. PMCID: PMC3875099. 